\title{
VARIANTS OF RECOGNITION PROBLEMS FOR MODULAR FORMS
}

\author{
E. KOWALSKI
}

\begin{abstract}
We consider the problem of distinguishing two modular forms, or two elliptic curves, by looking at the coefficients of their $L$-functions for small primes (compared to their conductor). Using analytic methods based on large-sieve type inequalities we give various upper bounds on the number of forms having the first few coefficients equal to those of a fixed one. In addition, we consider similar questions of "recognizing" symmetric squares and CM forms from the behavior of small primes.
\end{abstract}

\section{INTRODUCTION}

This paper is an addition to [DK], together with some variations on the problem considered there. It was motivated by discussions with F. Brumley who recently approached this question using a very nice method based on density theorems for zeros of $L$-functions.

Recall the setting, which generalizes the classical question of the least quadratic non-residue modulo a prime $p$ : given two primitive holomorphic cusp forms $f, g$ of the same weight $k \geqslant 1$ and nebentypus $\varepsilon$, with conductors $q(f), q(g)$ respectively, how large should $M$ be (in terms of the parameters described) so that the equality

for all primes $p \leqslant M$ implies that $f=g$ ? Here

$$
\lambda_{f}(p)=\lambda_{g}(p)
$$

$$
f(z)=\sum_{n \geqslant 1} \lambda_{f}(n) n^{(k-1) / 2} e(n z), \quad g(z)=\sum_{n \geqslant 1} \lambda_{g}(n) n^{(k-1) / 2} e(n z)
$$

are the Fourier expansions of $f$ and $g$, or in other words $\lambda_{f}(n)$ is the $n$-th Hecke eigenvalue for $f$.

The Generalized Riemann Hypothesis for Rankin-Selberg $L$-functions shows that one can take $M=$ $C(\log k q(f) q(g))^{2}$, where $C>0$ is some absolute (and effective) constant (see e.g. [IK, Prop. 5.22]).

The following is a reformulation of the main result of $[\mathrm{DK}]$ :

Theorem 1.1. Let $\alpha>0$ be any positive number and let $Q \geqslant 2$. For any primitive form $f$ of weight $k$ and conductor $q \leqslant Q$, the number $N$ of primitive non-CM modular forms $g$ of weight $k$ with conductor $q(g) \leqslant Q$ such that

$$
\lambda_{g}(p)=\lambda_{f}(p) \text { for } p \leqslant(\log Q)^{\alpha}
$$

is bounded by

$$
N \ll Q^{10 \alpha^{-1}+1 / 2+\varepsilon}
$$

for any $\varepsilon>0$, the implied constant depending only on $\alpha$ and $\varepsilon$.

Since the number of possible forms $g$ is $\gg Q^{2}$, it follows that for $\alpha>10$ the probability of $g$ satisfying (1) tends to 0 as $Q \rightarrow+\infty$. However, the exponent $\frac{1}{2}$ is unsatisfactory as it prevents the same conclusion to be reached if we look only for $g$ in a sub-family with less than about $Q^{1 / 2}$ elements of conductor $\leqslant Q$. In $[\mathrm{DK}]$, it is also shown that this exponent can be removed when looking at families with $q$ squarefree.

In [DK], this exponent arises from the need to use the symmetric square $L$-functions when the Fourier coefficients considered in (1) are "too small", so that one ends up counting not really the modular forms $g$ but their symmetric squares. As shown by Ramakrishnan in the Appendix to [DK], we have $\mathrm{Sym}^{2} f=\mathrm{Sym}^{2} g$ if and only if $f=g \otimes \chi$ for some primitive real character $\chi$ (the twist $g \otimes \chi$ is taken to be the primitive form associated to the modular form with Fourier coefficients $\lambda_{g}(n) \chi(n) n^{(k-1) / 2}$, which may be of smaller level). Thus the exponent $1 / 2$ arose by simply counting the possible number of characters $\chi$ such that the twist has conductor $\leqslant Q$.

In the next section we improve slightly the analysis of this case, removing the exponent $1 / 2$, but at the cost of introducing a dependency on $f$ in the final estimate. We will see that it does not seem

1991 Mathematics Subject Classification. Primary 11F12, 11F30; Secondary 11G05. 
easy to remove this dependency in general. ${ }^{1}$ However in Section 3, we get some uniform results for elliptic curves, using some cute arguments on group representations. In Section 4, we discuss two new variants: distinguishing symmetric squares from small primes, and CM forms from small primes inert in a quadratic field.

Notation. In general we denote by $\lambda_{f}(p)$ the Hecke eigenvalues for a primitive automorphic form, normalized so that the critical line for $L(f, s)$ is $\operatorname{Re}(s)=\frac{1}{2}$, independently of the weight. If $f$ is associated to an elliptic curve $E / \mathbf{Q}$, we write $a_{E}(p)$ for the Fourier coefficients so $a_{E}(p)=\sqrt{p} \lambda_{f}(p)$. We denote by $q(f)$ the conductor of a primitive form, and by $q(\chi)$ that of a Dirichlet character.

Finally, the notation $f \ll g$ and $f=O(g)$ are synonymous for us and refer to bounds for all $x$ in an explicitly specified set $X$ (sometimes clear from the context), with a well-defined implied constant.

\section{ReCOGNIZING A FIXED FORM}

Instead of fixing a form, it is maybe clearer to formulate the result as a multiplicity bound for a generic sequence of would-be eigenvalues. This viewpoint is inspired by that taken by Sarnak [Sa].

Proposition 2.1. Let $\Lambda=(\lambda(p))$ be a fixed sequence of complex numbers indexed by prime numbers. For any $\alpha>0$, any $Q \geqslant 2$, the number $N$ of primitive modular forms $g$ of weight 2 with conductor $q(g) \leqslant Q$ such that

$$
\lambda_{g}(p)=\lambda(p) \text { for } p \leqslant(\log Q)^{\alpha}
$$

is bounded by

$$
N \ll Q^{12 \alpha^{-1}+\varepsilon}
$$

for any $\varepsilon>0$, the implied constant depending on $\varepsilon$ and $\Lambda$.

We first state a slightly more precise version of the counting lemma that was used in [DK] and will be used repeatedly here.

Lemma 2.2. Let $Q \geqslant 2, \alpha>2, \beta>0, q \geqslant 1$. Let $m \geqslant 1$ be maximal such that $(\log Q)^{m \alpha} \leqslant Q^{\beta}$. Let $\mathcal{P}$ be a set of primes $p \leqslant(\log Q)^{\alpha}$ such that

$$
|\mathcal{P}| \geqslant \delta \frac{(\log Q)^{\alpha}}{\log \{\log Q\}^{\alpha}} .
$$

Then the number $N$ of integers $n \leqslant Q^{\beta}$ such that $n$ is squarefree, has $m$ prime factors, all of which belong to $\mathcal{P}$, satisfies

$$
Q^{\beta-\beta \alpha^{-1}-\varepsilon} \ll N \ll Q^{\beta-\beta \alpha^{-1}+\varepsilon}
$$

for any $\varepsilon>0$, the implied constants depending only on $\delta$, $\alpha$ and $\varepsilon$.

Proof. We have by Stirling's formula

$$
N=\left(\begin{array}{c}
\left|\mathcal{P}_{q}\right| \\
m
\end{array}\right) \gg m^{-1 / 2}\left(\frac{\left|\mathcal{P}_{q}\right|}{m}\right)^{m}
$$

with an absolute implied constant, where $\mathcal{P}_{q}$ is the set of primes $p \in \mathcal{P}$ not dividing $q$ (use $m^{2} \leqslant N$ ). We have

$$
\left|\mathcal{P}_{q}\right| \gg \frac{(\log Q)^{\alpha}}{\log \{\log Q\}^{\alpha}}
$$

by (2), the implied constant depending only on $\delta$ since $q$ has $\ll \log \log Q$ prime factors.

Hence we see that

$$
N \gg m^{-1 / 2}\left(\frac{(\log Q)^{\alpha}}{\log \left\{(\log Q)^{\alpha}\right\}} \frac{\alpha \log \log Q}{\beta \log Q}\right)^{m} \gg Q^{-\varepsilon}\left((\log Q)^{\alpha-1}\right)^{\frac{\beta(\log Q)}{(\alpha \log \log Q)}-1} \gg Q^{\beta \frac{\alpha-1}{\alpha}-\varepsilon} .
$$

On the other hand, Stirling's formula again yields similarly

$$
N \leqslant\left(\begin{array}{c}
|\mathcal{P}| \\
m
\end{array}\right) \ll\left(\frac{|\mathcal{P}|}{m}\right)^{m} \ll Q^{\beta \frac{\alpha-1}{\alpha}+\varepsilon} .
$$

\footnotetext{
${ }^{1}$ Note that also in the best individual and unconditional bounds currently known, e.g. in [KMV], [Ric] one also has to fix the form to which the others are compared.
} 
Proof of Proposition 2.1. Let $S$ be the set of forms $g$ that is being counted. One can assume $S$ not empty. The argument of $[\mathrm{DK}]$ gives the estimate

$$
N^{\prime} \ll Q^{10 \alpha^{-1}+\varepsilon}
$$

for the number $N^{\prime}$ of symmetric squares of the forms $g \in S$, and by Ramakrishnan's theorem, two elements $g_{1}, g_{2}$ of $S$ have the same symmetric square if and only if $g_{1}=g_{2} \otimes \chi$ for some real primitive quadratic character $\chi$.

Fix a form $g \in S$ with symmetric square having maximal multiplicity. For any $g_{1} \in S$ with the same symmetric square as $g$, we have $g_{1}=g \otimes \chi$ for some $\chi$ hence

$$
\lambda_{g_{1}}(p)=\chi(p) \lambda_{g}(p)
$$

for any $p$ coprime with $q\left(g_{1}\right)$ and $q(g)$. Using the assumption it follows that

$$
\lambda(p)=\chi(p) \lambda(p)
$$

for $p \leqslant(\log Q)^{\alpha}$ such that $\left(p, q\left(g_{1}\right) q(g)\right)=1$, so that either $\lambda(p)=0$ or $\chi(p)=1$ for those primes.

By results of Serre [Se1], the set of primes $p$ for which $\lambda_{g}(p) \neq 0$ has positive density $\geqslant \frac{1}{2}$ (in fact, density 1 if $g$ is not a CM form, and density $\frac{1}{2}$ if $g$ is a CM form). Since $S \neq \emptyset$ by assumption, this means that we have

$$
|\{p \leqslant X \mid \lambda(p) \neq 0\}| \gg \frac{X}{\log X}
$$

for $X=(\log Q)^{\alpha}$, the implied constant depending on $\Lambda$. Let $\mathcal{P}=\left\{p \leqslant(\log Q)^{\alpha} \mid \lambda(p) \neq 0\right\}$ be this set of primes. For any $\chi$ as above we have $\chi(p)=1$ for $p \in \mathcal{P}$ coprime with $q(g) q(\chi)$.

To estimate the number of $\chi$, we are therefore led back to Linnik's original problem, precisely to a simple variant since the set of primes involved is not the initial segment. For any primitive Dirichlet character $\psi$, write

$$
L(\psi)=\sum_{n \leqslant Q^{2}} a_{n} \psi(n)
$$

where $a_{n}$ is the characteristic function of the set $T$ of integers $n \leqslant Q^{2}$ such that $n$ is squarefree, has a fixed number $m$ of prime factors, and $p \mid n$ implies $p \in \mathcal{P}$ and $(p, q(g))=1$. We have moreover

$$
L(\chi)=|\{n \in T \mid(n, q(\chi))=1\}|
$$

if $g \otimes \chi \in S$. By Lemma 2.2 twice (with the same $m$, but with different $q$ ), if $m$ is suitably chosen, we have

$$
|T| \geqslant L(\chi) \gg Q^{2\left(1-\alpha^{-1}\right)-\varepsilon},|T| \ll Q^{2\left(1-\alpha^{-1}\right)+\varepsilon} \text {, so } L(\chi) \gg|T|^{1-\varepsilon}
$$

for any $\varepsilon>0$, the implied constants depending on $\alpha, \varepsilon$ and $\Lambda$ (not on $\chi$ ).

On the other hand, by positivity and the multiplicative large sieve inequality we have

$$
|T|^{2-\varepsilon}|\{\chi \mid g \otimes \chi \in S\}| \leqslant \sum_{q \leqslant Q} \sum_{\chi(\bmod q)}^{*}|L(\chi)|^{2} \leqslant Q^{2} \sum_{n \leqslant Q^{2}}\left|a_{n}\right|^{2} \leqslant Q^{2}|T|
$$

so the number of characters is $\ll Q^{2 \alpha^{-1}+\varepsilon}$ for any $\varepsilon>0$, the implied constant depending on $\alpha, \varepsilon$ and $\Lambda$.

This bounds the maximal possible multiplicity of the symmetric square, hence

$$
N \ll N^{\prime} Q^{2 \alpha^{-1}+\varepsilon} \ll Q^{12 \alpha^{-1}+\varepsilon}
$$

for any $\varepsilon>0$, the implied constant depending on $\Lambda, \alpha$ and $\varepsilon$.

To remove the dependency of the result on the sequence $\Lambda$ amounts to giving a uniform version of (3) for $\lambda(p)=\lambda_{f}(p)$. This is certainly difficult because the primes involved are very small, so even the existence of one $p \leqslant(\log Q)^{\alpha}$ with $\lambda_{f}(p) \neq 0$ is an extremely strong result for $f$ of conductor about $Q$. And since, if a form exists with many zero coefficients, then many twists may have the same symmetric square and the same first Fourier coefficients, one cannot expect to deal with $g$ on average only.

The case of Maass forms, also considered by Brumley, can not be treated as above because an analogue of (3) is not known in this case; one only knows that there are $\gg_{g} x / \log x$ values of $n \leqslant x$ such that $\lambda_{f}(n) \neq 0$ (see Proposition 3 of $[\mathrm{KRW}]$ ). 


\section{RECOGNIZING ELLIPTIC CURVES}

In the case of elliptic curves, one can derive a strong uniform version on the assumption of the Riemann Hypothesis, not for all $L$-functions (since GRH for Rankin-Selberg $L$-functions gives more than what we want!), but for real characters and for holomorphic modular forms of weight 1 (with solvable image, if one wishes). Without GRH, a much weaker uniform result follows by the standard zero-free regions:

Proposition 3.1. Let $\alpha>0, Q \geqslant 2$ and let $X$ be a set with maximal cardinality of isogeny classes of elliptic curves $E / \mathbf{Q}$ with conductors $\leqslant Q$ such that

$$
a_{E}(p)=a_{F}(p) \text { for } E, F \in X, p \leqslant(\log Q)^{\alpha} .
$$

(1) Assuming GRH for Dirichlet L-functions of real characters and holomorphic modular forms of weight 1, we have

$$
|X| \ll Q^{12 \alpha^{-1}+\varepsilon}
$$

for any $\varepsilon>0$, the implied constant depending on $\alpha$ and $\varepsilon$ only.

(2) Unconditionally, we have

$$
|X| \ll Q^{12 \alpha^{-1}+\varepsilon}
$$

provided $X$ contains at least one curve with conductor $\leqslant \log \{\log Q\}^{\alpha}$. The implied constant depends on $\alpha$ and $\varepsilon$ only.

We need a lemma (probably well-known) to bound the conductors of some Artin $L$-functions. In what follows, we denote by $G_{\mathbf{Q}}$ the Galois group of a separable closure of $\mathbf{Q}$.

Lemma 3.2. Let $E / \mathbf{Q}$ be an elliptic curve, $\ell$ be a prime number, $\rho_{\ell}: G_{\mathbf{Q}} \rightarrow G L(2, \mathbf{Z} / \ell \mathbf{Z})$ the Galois representation given by the action on the $\ell$-torsion points of $E$. For any irreducible linear representation $\pi: G L(2, \mathbf{Z} / \ell \mathbf{Z}) \rightarrow G L(m, \mathbf{C})$, the Artin conductor of the representation $\pi \circ \rho_{\ell} i s \leqslant c(\ell) q^{m}$, where $q$ is the conductor of $E$ and $c(\ell) \geqslant 1$ is an integer depending only on $\ell$.

Proof. For any fixed prime $p$, notice that for any real number $v \geqslant-1$, the upper-numbering ramification group $I_{p}^{v}$ (normalized as in [DDT, $\S 2.1$ ], so $I_{p}^{v}$ is the inertia group at $p$ for $v \leqslant 0$ ) acts trivially on $\rho_{\ell}$ and on $\pi \circ \rho_{\ell}$ if it acts trivially on the $\ell$-torsion points of $E$. For $p \neq \ell$, the exponent of the Artin conductor of $\pi \circ \rho_{\ell}$ at $p$ is given by

$$
v_{p}\left(\pi \circ \rho_{\ell}\right)=\int_{-1}^{+\infty} \operatorname{codim}\left(\pi \circ \rho_{\ell}\right)^{I_{p}^{v}} d v
$$

(see e.g. [DDT, p. 49]). Let $V$ be such that $I_{p}^{v}$ acts trivially on $E[\ell]$ for $v>V$ and non-trivially for $v<V$. Then we have

$$
v_{p}\left(\pi \circ \rho_{\ell}\right)=\int_{-1}^{V} \operatorname{codim}\left(\pi \circ \rho_{\ell}\right)^{I_{p}^{v}} d v \leqslant m(V+1)
$$

and similarly the exponent of the conductor for the representation modulo $\ell$ is

$$
v_{p}\left(\rho_{\ell}\right)=\int_{-1}^{V} \operatorname{codim}\left(\rho_{\ell}\right)^{I_{p}^{v}} d v \geqslant V+1,
$$

since $\operatorname{codim}\left(\rho_{\ell}\right)^{I_{p}^{v}}$ must be $\geqslant 1$ if the action is non-trivial. Since $p^{v_{p}\left(\rho_{\ell}\right)} \mid q$ (see e.g. [DDT, Lemma 2.7, Remark 2.14]), we obtain the result at $p$.

For $p=\ell$, let $K_{\ell}=\mathbf{Q}(E[\ell])$ be the field generated by coordinates of $\ell$-torsion points of $E$ and $n=\left[K_{\ell}: \mathbf{Q}\right]$. The exponent of $\ell$ in the discriminant of $K_{\ell} / \mathbf{Q}$ is the same as the exponent of the conductor of the regular representation $\omega: G_{\mathbf{Q}} \rightarrow \mathbf{C}\left[\mathrm{Gal}\left(K_{\ell} / \mathbf{Q}\right)\right] \simeq G L(n, \mathbf{C})$ (see e.g. [Se3, VI.3, Cor. 1]). Hence as above it is given by

$$
v_{\ell}\left(K_{\ell} / \mathbf{Q}\right)=\int_{-1}^{+\infty} \operatorname{codim}(\omega)^{I_{p}^{v}} d v .
$$

Let $V$ be as above for $\ell$, so also $I_{p}^{v}$ acts trivially on $\omega$ for $v>V$. We have

$$
\begin{aligned}
& v_{\ell}\left(\pi \circ \rho_{\ell}\right)=\int_{-1}^{V} \operatorname{codim}\left(\pi \circ \rho_{\ell}\right)^{I_{\ell}^{v}} d v \leqslant m(V+1) \\
& v_{\ell}\left(K_{\ell} / \mathbf{Q}\right)=\int_{-1}^{V} \operatorname{codim}(\omega)^{I_{p}^{v}} d v \geqslant V+1 .
\end{aligned}
$$


Hence to prove the desired result it is enough to find an upper bound for $v_{\ell}\left(K_{\ell} / \mathbf{Q}\right)$ which is independent of $E$. Such a bound follows, for instance, from the fact that the degree of $K_{\ell}$ is bounded independently of $E$, and from universal bounds for the discriminant of extensions of local fields of bounded degree (see, e.g., [Se1, Cor. to Prop. 2]).

Remark 3.3. For our application, one could also simply bound the conductor of $\pi \circ \rho_{\ell}$ by the discriminant of $K_{\ell}$ and apply a general bound like [Se1, Prop. 6].

Although we will use only the case $q=3$ of the next lemma, it is nice enough to state generally.

Lemma 3.4. Let $\mathbf{F}_{q}$ be a field with $q$ elements of characteristic $\neq 2$. Let $N$ be the set of elements in $G L\left(2, \mathbf{F}_{q}\right)$ which are not diagonalizable over $\overline{\mathbf{F}}_{q}, f_{N}$ the characteristic function of $N$. Then we have

$$
q f_{N}=1+\chi \circ \operatorname{det}+\sum_{\psi^{2} \neq 1} \chi_{\psi, \psi^{-1}}-\sum_{\varphi \mid \mathbf{F}_{q}=1} \chi_{\varphi}
$$

where $\chi$ is the quadratic character of $\mathbf{F}_{q}, \psi$ runs over characters of $\mathbf{F}_{q}^{\times}$and $\chi_{\psi, \psi^{-1}}$ is the character of the associated irreducible representations of degree $q+1, \varphi$ runs over characters of $\mathbf{F}_{q^{2}}^{\times}$with $\varphi^{q} \neq \varphi$ and $\chi_{\varphi}$ is the character of the associated irreducible representation of degree $q-1$; see the discussion below for details.

Proof. The set $N$ is the union of the $q-1$ conjugacy classes of the elements

$$
n_{x}=\left(\begin{array}{ll}
x & 1 \\
0 & x
\end{array}\right)
$$

with $x \neq 0$, each of which has $q^{2}-1$ elements. To decompose $f_{N}$ in terms of characters, we need only look at the corresponding column of the character table, which we quote with the notation of $[\mathrm{FH}, \mathrm{p}$. 70] (transposed for easier reading, and with the degree of the representations for reference):

\begin{tabular}{c|c|c|c|c} 
& $U_{\alpha}$ & $V_{\alpha}$ & $W_{\alpha, \beta}$ & $X_{\varphi}$ \\
\hline degree & 1 & $q$ & $q+1$ & $q-1$ \\
$n_{x}$ & $\alpha\left(x^{2}\right)$ & 0 & $\alpha(x) \beta(x)$ & $-\varphi(x)$
\end{tabular}

$\left(U_{\alpha}\right.$ is the character $\alpha(\operatorname{det}(x)), V_{\alpha}$ is the irreducible component of degree $q$ of the permutation representation on $\mathbf{P}^{1}\left(\mathbf{F}_{q}\right)$, twisted by $\alpha, W_{\alpha, \beta}$ is induced from the character $(\alpha, \beta)$ of the Borel subgroup (up to permutation) with $\alpha \neq \beta$, and $X_{\varphi}$, for $\varphi$ a character of $\mathbf{F}_{q^{2}}^{\times}$such that $\varphi^{q} \neq \varphi$, is the (a priori virtual) representation $\left.V_{1} \otimes W_{1, \varphi}-W_{1, \varphi}-\operatorname{Ind}(\varphi)\right)$.

The obvious point now is that for each irreducible character $\chi, \chi$ restricted to $n_{x} \in N$ is, as function of $x$, a constant multiple of some multiplicative character of $\mathbf{F}_{q}^{\times}$. Hence the scalar product $\left\langle f_{N}, \chi\right\rangle$ vanishes by orthogonality unless the corresponding character is trivial on $\mathbf{F}_{q}^{\times}$. This immediately implies (5) by inspection.

Remark 3.5. In particular, among the $q^{2}-1$ irreducible representations of $G L\left(2, \mathbf{F}_{q}\right)$, only $q$ occur in the decomposition of $f_{N}$. One can prove more conceptually that characters restricted to $N$ are multiples of multiplicative characters, as pointed out by D. Bump: the matrix $n_{x}$ is conjugate to

$$
\left(\begin{array}{ll}
x & x \\
0 & x
\end{array}\right)=\left(\begin{array}{ll}
x & 0 \\
0 & x
\end{array}\right)\left(\begin{array}{ll}
1 & 1 \\
0 & 1
\end{array}\right)
$$

hence, if $\psi$ is the central character, we have

$$
\chi\left(n_{x}\right)=c \psi(x) \text { with } c=\chi\left(\left(\begin{array}{ll}
1 & 1 \\
0 & 1
\end{array}\right)\right) .
$$

Proof of Proposition 3.1. By modularity of elliptic curves and the reasoning at the beginning of the proof of Proposition 2.1, it suffices to prove a uniform lower bound for the number of primes $\leqslant(\log Q)^{\alpha}$ where $a_{E}(p)=0$.

For any prime $\ell$, the Galois representation $\rho_{\ell}: G_{\mathbf{Q}} \rightarrow G L(2, \mathbf{Z} / \ell \mathbf{Z})$ on $\ell$-torsion points satisfies $a_{E}(p) \equiv \operatorname{Tr} \rho_{\ell}\left(\operatorname{Fr}_{p}\right)(\bmod \ell)$ for any unramified prime $p \neq \ell$. In particular, if $\rho_{\ell}\left(\operatorname{Fr}_{p}\right)$ is in the set of conjugacy classes with non-zero trace in $\mathbf{Z} / \ell \mathbf{Z}$, we have $a_{E}(p) \neq 0$.

If $E$ does not have non-trivial rational 2-torsion points, one can use $\ell=2$, but if $E[2](\mathbf{Q}) \neq 0$ we have $a_{E}(p) \equiv 0(\bmod 2)$ for any odd prime. To avoid considering an unnecessary case, we take $\ell=3$.

We distinguish two cases, depending on whether $G_{3}=\rho_{3}\left(G_{\mathbf{Q}}\right) \subset G L(2, \mathbf{Z} / 3 \mathbf{Z})$ has order divisible by 3 or not. If $G_{3}$ has order divisible by 3 , it must contain elements of the set $N$ described in Lemma 3.4 
for $q=3$. For any $x \in N$, we have $\operatorname{Tr} x= \pm 1 \neq 0$, so any prime $p$ for which $\rho_{3}\left(\operatorname{Fr}_{p}\right) \in G_{3} \cap N$ satisfies $a_{E}(p) \neq 0$, and we know a priori from the Chebotarev density theorem that this set of primes has positive density.

By Lemma 3.4, all characters of $\mathbf{F}_{3}^{\times}$being quadratic, the characteristic function $f_{N}$ of $N$ is given by

$$
3 f_{N}=1+\operatorname{det}-\chi_{2},
$$

where 1 denotes the character of the trivial representation, det is the determinant quadratic character, and $\chi_{2}$ is the character of an irreducible representation of degree 2 (precisely of $X_{\varphi}$ where $\varphi$ is the character of $\mathbf{F}_{9}^{\times}=\mathbf{F}_{3}[X]^{\times}$, with $X^{2}=-1$, that maps the primitive root $1+X \in \mathbf{F}_{9}^{\times}$to $\left.i \in \mathbf{C}\right)$.

It might be that $\chi_{2}$ restricted to $G_{3}$ is not irreducible, in which case it splits into a direct sum of two characters of degree 1 , say $\varepsilon_{1}, \varepsilon_{2}$. Since the set of primes we're counting has density $>0$ by assumption, the trivial representation occurs with coefficient $>0$ in the decomposition of $f_{N}$ so one has $\varepsilon_{i} \neq 1$ (see $(6))$.

The Artin $L$-functions of 1 , det (and $\varepsilon_{1}, \varepsilon_{2}$ possibly) are Dirichlet $L$-functions, and since $G_{3}$ is solvable, the Artin $L$-function of $\chi_{2}$ is the $L$-function of a primitive holomorphic cusp form of weight 1 by the Langlands-Tunnell Theorem. The conductors of all those $L$-functions are $\ll Q^{2}$ by Lemma 3.2 and we obtain

$$
\begin{aligned}
3 \sum_{\substack{p \leqslant X \\
a_{E}(p) \neq 0}} \log p & \geqslant 3 \sum_{p \leqslant X} f_{N}\left(\rho_{3}\left(\operatorname{Fr}_{p}\right)\right) \log p \\
& =\sum_{p \leqslant X} \log p+\sum_{p \leqslant X} \operatorname{det}\left(\rho_{3}\left(\operatorname{Fr}_{p}\right)\right) \log p-\sum_{p \leqslant X} \chi_{2}\left(\rho_{3}\left(\operatorname{Fr}_{p}\right)\right) \log p+O(1) .
\end{aligned}
$$

By GRH for 1 , det, $\chi_{2}$ (or $\varepsilon_{1}, \varepsilon_{2}$ ) we get for $X \geqslant 2$ that

$$
3 \sum_{\substack{p \leqslant X \\ a_{E}(p) \neq 0}} \log p \geqslant X+O\left(\sqrt{X}(\log Q X)^{2}\right),
$$

with an absolute implied constant (see e.g. [IK, Th. 5.15]). Taking $X=(\log Q)^{\alpha}$, it follows by partial summation that for $\alpha>5$ we have

$$
\left|\left\{p \leqslant(\log Q)^{\alpha} \mid a_{E}(p) \neq 0\right\}\right| \gg \frac{(\log Q)^{\alpha}}{\log \left\{(\log Q)^{\alpha}\right\}}
$$

with an absolute implied constant, which is the required uniform version of (3). If $\alpha \leqslant 5$, the statement of Proposition 3.1 is trivial in any case.

If $G_{3}$ has order divisible by 3 (which means that the discriminant of $E$ is a cube, see e.g. [Se2, p. 305]), it only contains semisimple elements and its order is at most 16. It is either abelian or dihedral and its irreducible representations are thus of degree 1 or 2 . Decomposing the characteristic function of the identity (with trace $-1 \neq 0$ ) in characters, one gets a lower bound like $(7)$ using $L$-functions of degree 1 or 2 again.

We now come to what can be proved without GRH. For any primitive Dirichlet character $\chi$ modulo $q$ we have

$$
\sum_{p \leqslant X} \chi(p) \log p=\delta(\chi) X+O\left(\frac{\sqrt{q} X}{(\log X)^{A}}\right)
$$

for $X \geqslant 2$ and $A>0$, the implied constant depending on $A$ only. For a holomorphic cusp form $f$ of weight 1 with conductor $q$ we have

$$
\sum_{p \leqslant X} \lambda_{f}(p) \log p \ll \frac{\sqrt{q} X}{(\log X)^{A}}
$$

for $X \geqslant 2$ and $A>0$, the implied constant depending on $A$ only (see e.g. [IK, Cor. 5.29, Th. 5.40]; by a result of Stark [St] for Artin $L$-functions of degree $>1$, or of Hoffstein and Ramakrishnan [HR] for cusp forms, the $L$-function of the forms that occur here do not have exceptional zeros). Denoting by $q$ the conductor of $E$ and taking again the case of $\ell=3$ with $G_{3}$ of order divisible by 3, we have now

$$
3 \sum_{\substack{p \leqslant X \\ a_{E}(p) \neq 0}} \log p \geqslant X+O\left(\frac{q X}{(\log X)^{A}}\right) .
$$


by (6) and the upper bound for the conductors of the $L$-functions for $\operatorname{det}$ and $\chi_{2}$. Take $A=2$. If $X=(\log Q)^{\alpha}$ and $q \leqslant \log X$, we obtain

$$
\left|\left\{p \leqslant(\log Q)^{\alpha} \mid a_{E}(p) \neq 0\right\}\right| \gg \frac{(\log Q)^{\alpha}}{\log \left\{(\log Q)^{\alpha}\right\}}
$$

where the implied constant is absolute. The other case is obviously similar.

\section{VARIATIONS: RECOGNIZING SYMMETRIC SQUARES AND CM FORMS}

The theorem of Ramakrishnan characterizing the non-injectivity of the symmetric square on modular forms suggests the following problem: assume that $f$ and $g$ are primitive forms (with same weight $k$ ) and that $\lambda_{f}\left(p^{2}\right)=\lambda_{g}\left(p^{2}\right)$ for $p \leqslant M$. How large should $M$ be so that $\mathrm{Sym}^{2} f=\mathrm{Sym}^{2} g$, or in other words, so that $f=g \otimes \chi$ for some real primitive character $\chi$ ?

By GRH for the Rankin-Selberg $L$-functions $L\left(\operatorname{Sym}^{2} f \otimes \operatorname{Sym}^{2} g, s\right)$ and $L\left(\operatorname{Sym}^{2} f \otimes \operatorname{Sym}^{2} f, s\right)$, it follows that if $f$ and $g$ are not CM forms, the conclusion $\mathrm{Sym}^{2} f=\mathrm{Sym}^{2} g$ follows for $M=C(\log k q(f) q(g))^{2}$ for some absolute constant $C>0$. (In the CM case, one gets a bound of same size by working over the $\mathrm{CM}$ field, using the corresponding Hecke characters).

Using the Linnik method we get:

Theorem 4.1. Let $\alpha>0$ be any positive number and let $Q \geqslant 2$. For any weight 2 primitive form $f$ of conductor $q \leqslant Q$, the number $N$ of symmetric squares of primitive non-CM modular forms $g$ of weight 2 and conductor $q(g) \leqslant Q$ such that

$$
\lambda_{g}\left(p^{2}\right)=\lambda_{f}\left(p^{2}\right) \text { for } p \leqslant(\log Q)^{\alpha}
$$

is bounded by

$$
N \ll Q^{24 \alpha^{-1}+\varepsilon}
$$

for any $\varepsilon>0$, the implied constant depending only on $\alpha$ and $\varepsilon$.

There is no extraneous exponent here, but as the proof will clearly show this is because we fix the nebentypus to be trivial. The proof follows [DK] again, but this time we must use a mean-value estimate for the symmetric fourth power $L$-functions (available from [DK] thanks to Kim's result [K] that for a non-CM modular form $g$, its fourth symmetric power $\mathrm{Sym}^{4} \mathrm{~g}$ is a cuspidal automorphic representation on $G L(5)$ with conductor $\left.\leqslant q^{4} \leqslant Q^{4}\right)$ if the $\lambda_{f}\left(p^{2}\right)$ are too small for small $p$. An issue of multiplicity arises for the symmetric fourth power. The simplest case, which suffices our purpose, is as follows:

Lemma 4.2. For any two holomorphic primitive forms $f$ and $g$ of weight $\geqslant 2$ and trivial nebentypus, we have $\mathrm{Sym}^{4} f=\mathrm{Sym}^{4} g$ if and only if $\mathrm{Sym}^{2} f=\mathrm{Sym}^{2} g$.

This follows either from Proposition 5.1 of [CM], or from more general results of Rajan [R] on recovering $\ell$-adic representations. This case is an easy application of Serre's $\ell$-adic methods, and the non-expert reader is invited to see it as an interesting exercise (it boils down to showing that $\lambda_{f}\left(p^{2}\right)=1-\lambda_{g}\left(p^{2}\right)$ can not occur very often). Note that other cases of this lemma are a little bit different. With non-trivial nebentypus, further multiplicity comes trivially from quartic twists $f \otimes \chi_{4}$, where $\chi_{4}$ is of order 4 exactly. More interestingly, for forms of weight 1 there is a different source of multiplicity: if $f$ corresponds to an icosahedral Galois representation, then $f$ has a Galois conjugate $f^{\tau}$ (where $\tau$ basically is the non-trivial automorphism of $\mathbf{Q}(\sqrt{5}) / \mathbf{Q}$ ), with $f^{\tau}$ not a quartic twist of $f$, and $\operatorname{Sym}^{4} f^{\tau}=\operatorname{Sym}^{4} f$. (The proof above fails because $(1-\sqrt{5}) / 2=1-(1+\sqrt{5}) / 2 \ldots)$

This in particular means that the corresponding statement for Maass forms is certainly quite deep as the Langlands-type correspondance is not known to exist for even icosahedral representations.

Proof. Let $p$ be an unramified prime for $f$, and let $\alpha$ and $\beta$ be the usual local roots of $f$ at $p$, so that $\alpha+\beta=\lambda_{f}(p)$ and $\alpha \beta=1$. We have $\lambda_{f}\left(p^{2}\right)=\alpha^{2}+\beta^{2}+1$ and

$$
\lambda_{f}\left(p^{4}\right)=\alpha^{4}+\alpha^{2}+1+\beta^{2}+\beta^{4}=\left(\alpha^{2}+\beta^{2}+1\right)^{2}-\alpha^{2}-\beta^{2}-2=\lambda_{f}\left(p^{2}\right)^{2}-\lambda_{f}\left(p^{2}\right)-1 .
$$

In particular if $\left|\lambda_{f}\left(p^{2}\right)\right|<\frac{1}{2}$, we have $\left|\lambda_{f}\left(p^{4}\right)\right| \geqslant 1 / 4$. If more than half the primes $p \leqslant(\log Q)^{\alpha}$ satisfy $\left|\lambda_{f}\left(p^{2}\right)\right|<\frac{1}{2}$, then we derive

$$
N \ll Q^{10 \alpha^{-1}+\varepsilon}
$$

from the argument of [DK]. 
Otherwise we have $\left|\lambda_{f}\left(p^{4}\right)\right| \geqslant 1 / 4$ for at least half the primes $\leqslant(\log Q)^{\alpha}$. For the usual parameters $X \geqslant 2$ and $m \geqslant 1$ to be chosen later, denote

$$
L(g)=\sum_{n \leqslant X} b_{n} \lambda_{g}\left(n^{4}\right)
$$

where $b_{n}=a_{n} \lambda_{f}\left(n^{4}\right)$, and $a_{n}$ is the characteristic function of those integers $n \leqslant X$ such that $n$ is squarefree and has $m$ prime factors, all unramified for $f$, with $\left|\lambda_{f}\left(p^{4}\right)\right| \geqslant 1 / 4$. For any $g$ where $\operatorname{Sym}^{2} g$ contributes to $N$ we have

$$
L(g) \geqslant \frac{1}{16^{m}} \sum_{n \leqslant X} a_{n}
$$

By positivity we have

$$
\frac{N}{256^{m}}\left(\sum_{n \leqslant X} a_{n}\right)^{2} \leqslant \sum_{q \leqslant Q} \sum_{g}|L(g)|^{2}
$$

where $g$ runs over non-CM forms of weight 2 and conductor $q$. Let

$$
L\left(\operatorname{Sym}^{4} g, s\right)=\sum_{n \geqslant 1} \nu_{g}(n) n^{-s}
$$

be the Dirichlet series expansion of the fourth symmetric power $L$-function of $f$. By the above properties of $\lambda_{f}\left(p^{4}\right)$ we can write

$$
\sum_{q \leqslant Q} \sum_{g}|L(g)|^{2}=\sum_{q \leqslant Q} \sum_{g}\left|\sum_{n \leqslant X} a_{n} \nu_{g}(n)\right|^{2} .
$$

By Kim's functoriality theorem and Theorem 4 of $[\mathrm{DK}]$, we have

$$
\sum_{q \leqslant Q} \sum_{\mathrm{Sym}^{4}}\left|\sum_{n \leqslant X} a_{n} \nu_{g}(n)\right|^{2} \ll X^{1+\varepsilon} \sum_{n \leqslant X}\left|a_{n}\right|^{2}=X^{1+\varepsilon} \sum_{n \leqslant X} a_{n}
$$

if $X>Q^{\beta}$ with $\beta>24$, for any $\varepsilon>0$, the implied constant depending on $\beta$ and $\varepsilon$. (The exponent 24 arises because the conductor is $\leqslant Q^{4}$, and the number of forms $\ll Q^{2}=Q^{4 / 2}$ so $d=1 / 2, n=5$, in the notation of loc. cit.)

Note the last sum is over the symmetric fourth powers of $g$. By Lemma 4.2, we can rewrite the sum over $\mathrm{Sym}^{4} g$ as one over $\mathrm{Sym}^{2} g$, which is precisely what we are counting. By Lemma 2.2, for suitable $m$ (maximal such that $(\log Q)^{m \alpha} \leqslant Q^{24}$ ), we have for any $\varepsilon>0$ the lower bound

$$
\sum_{n \leqslant X} a_{n} \gg Q^{24\left(1-\alpha^{-1}\right)-\varepsilon}
$$

when choosing $m$ maximal with $(\log Q)^{\alpha m} \leqslant Q^{\beta}, \beta>24$, the implied constant depending only on $\alpha$ and $\varepsilon$. Hence the result follows as before, $m$ being small enough that $256^{m} \ll X^{\varepsilon}$ for any $\varepsilon>0$.

The final problem we consider is motivated now by the result of Serre used in the proof of Proposition 2.1: let $k / \mathbf{Q}$ be a fixed quadratic field, and let $f$ be primitive form of weight $k \geqslant 2$; assume that $\lambda_{f}(p)=0$ for all $p \leqslant M$ which are inert in $k$. How large should $M$ be so that this implies that $f$ is a CM form?

Denote by $\chi$ the quadratic character associated with $k$ and by $D$ the discriminant of $k$. The assumption implies that $\lambda_{f}(p)=\chi(p) \lambda_{f}(p)=\lambda_{f \otimes \chi}(p)$ for all $p \leqslant M$ unramified in $k$. Hence by GRH again, it follows that $f=f \otimes \chi$ if $M \geqslant C(\log |D| q(f))^{2}$ with an absolute constant $C \geqslant 0$. Since this condition implies that $f$ is a CM form, this solves the problem.

Here is a Linnik-style result about this problem. Since the condition $\lambda_{f}(p)=0$ is stable by any twist by a character, it is natural to count the forms up to twist; fixing the nebentypus, this means up to quadratic twist (to put it differently, a twist of a CM form is itself a CM form, but the nebentypus changes except for quadratic twists). So it is natural to count only the number of possible symmetric squares.

Theorem 4.3. Let $\alpha>0$ be any positive number and let $Q \geqslant 2$. For any quadratic field $k / \mathbf{Q}$, the number $N$ of symmetric squares of primitive modular forms of weight 2 and conductor $\leqslant Q$ such

$$
\lambda_{f}(p)=0 \text { for } p \leqslant(\log Q)^{\alpha} \text { inert in } k
$$

is bounded by

$$
N \ll Q^{10 \alpha^{-1}+\varepsilon}
$$

for any $\varepsilon>0$, the implied constant depending only on $\alpha$ and $\varepsilon$. 
Proof. If $f$ satisfies (10), we have $\lambda_{f}\left(n^{2}\right)=1$ for all $n$ squarefree which is divisible only by primes $p \leqslant(\log Q)^{\alpha}$ which are inert in $k$, and unramified for $f$. Thus with

$$
L(f)=\sum_{n \leqslant X} a_{n} \lambda_{f}\left(n^{2}\right)
$$

where $a_{n}$ is the characteristic function of numbers of this type having exactly $m$ prime factors, we have for such $f$

$$
L(f)=\sum_{n \leqslant X} a_{n}
$$

and by positivity we have

$$
N\left(\sum_{n \leqslant X} a_{n}\right)^{2} \leqslant \sum_{f}^{*}|L(f)|^{2}
$$

where $\sum^{*}$ indicates that we sum over the distinct symmetric squares only. By the large-sieve type inequality for symmetric square $L$-functions, we have

$$
\sum_{f}^{*}|L(f)|^{2} \ll X^{1+\varepsilon} \sum_{n \leqslant X}\left|a_{n}\right|^{2}=X^{1+\varepsilon} \sum_{n \leqslant X} a_{n},
$$

if $X>Q^{\beta}$ for any $\beta>10$ and $\varepsilon>0$, the implied constant depending only on $\beta$ and $\varepsilon$.

To estimate the number of $n$ with $a_{n}=1$, we have the analogue of (3)

$$
\mid\{p \leqslant X \mid p \text { is inert in } k\} \mid \gg \frac{X}{\log X},
$$

for $X \geqslant 2$, the implied constant depending on $k$. Applying Lemma 2.2 to this situation (again for $m$ as described in the statement), this gives the bound $Q^{10 \alpha^{-1}+\varepsilon}$ for the number of symmetric squares.

\section{REFERENCES}

[CM] J. Cogdell and P. Michel: On the complex moments of symmetric power L-functions at s = 1, I.M.R.N 2004, no. 31, 1561-1617.

[DDT] H. Darmon, F. Diamond and M. Taylor: Fermat's Last Theorem, Current Developments in Math., International Press (1995), 1-154.

[DK] W. Duke and E. Kowalski: A problem of Linnik for elliptic curves and mean-value estimates for automorphic representations, Invent. math. 139 (2000), 1-39.

[FH] W. Fulton and J. Harris: Representation theory. A first course, GTM 129, Springer 1991.

[HR] J. Hoffstein and D. Ramakrishnan: Siegel zeros and cusp forms, I.M.R.N 1995, No.6, 279-308 (1995).

[IK] H. Iwaniec and E. Kowalski: Analytic Number Theory, A.M.S Colloquium Series 53, 2004.

[K] H. Kim: Functoriality for the exterior square of $\mathrm{GL}_{4}$ and the symmetric fourth of $\mathrm{GL}_{2}$, with appendix 1 by $\mathrm{D}$. Ramakrishnan and appendix 2 by H. Kim and P. Sarnak, J. Amer. Math. Soc. 16 (2003), no. 1, 139-183.

[KMV] E. Kowalski, P. Michel and J. VanderKam: Rankin-Selberg L-functions in the level aspect, Duke Math. J. 114 (2002), no. 1, 123-191.

[KRW] E. Kowalski, O. Robert and J. Wu: Small gaps in coefficients of L-functions and $\mathfrak{B}$-free numbers in small intervals, preprint (2003).

[R] C.S. Rajan: Recovering $\ell$-adic representations, preprint (2002).

[Ric] G. Ricotta: Zéros réels et taille des fonctions L de Rankin-Selberg par rapport au niveau, thèse de doctorat, Université Montpellier II (2004).

[Sa] P. Sarnak: letter to Zeev Rudnick.

[Se1] J-P. Serre: Quelques applications du théorème de densité de Chebotarev, Publ. Math. I.H.E.S 54 (1981), 123-201.

[Se2] J-P. Serre: Propriétés galoisiennes des points d'ordre fini des courbes elliptiques, Invent. math. 15 (1972), $259-331$.

[Se3] J-P. Serre: Corps locaux, 3ème ed., Hermann 1968.

[St] H. Stark: Some effective cases of the Brauer-Siegel theorem, Invent. math. 23 (1974), 135-152.

Université Bordeaux I - A2X, 351, cours de la Libération, 33405 Talence Cedex, France

E-mail address: emmanuel.kowalski@math.u-bordeaux1.fr 